\title{
Theory of proton coupled electron transfer reactions: Assessing the Born-Oppenheimer approximation for the proton motion using an analytically solvable model
}

\author{
Renhui Zheng, Yuanyuan Jing, Liping Chen, and Qiang Shi* \\ Beijing National Laboratory for Molecular Sciences, \\ State Key Laboratory for Structural Chemistry of Unstable and Stable Species, \\ Institute of Chemistry, Chinese Academy of Sciences, \\ Zhongguancun, Beijing 100190, China
}

\begin{abstract}
By employing an analytically solvable model including the Duschinsky rotation effect, we investigated the applicability of the commonly used Born-Oppenheimer (BO) approximation for separating the proton and proton donor-acceptor motions in theories of proton coupled electron transfer (PCET) reactions. Comparison with theories based on the BO approximation shows that, the BO approximation for the proton coordinate is generally valid while some further approximations may become inaccurate in certain range of parameters. We have also investigated the effect of vibrationally coherent tunneling in the case of small reorganization energy, and shown that it plays an important role on the rate constant and kinetic isotope effect.
\end{abstract}

*Electronic address: qshi@iccas.ac.cn 


\section{INTRODUCTION}

Proton coupled electron transfer (PCET) reactions are important in many chemical, biological and electro-chemical processes. [1 -5] We consider in this paper the case of concerted PCET reactions, where the proton and electron are transferred simultaneously with no stable intermediates. Theories of PCET reactions were developed over the past two decades. Cukier has proposed a theory for concerted PCET based on nonadiabatic transitions between multiple vibronic states, [6 8] which treats the proton coordinate as a high frequency intramolecular vibrational mode, and leads to rate constant expressions similar to the Bixon-Jortner model[9] for the electron transfer (ET) reactions.[10, 11] Hammesschiffer and coworkers have generalized this theory to include the environmental collective coordinate that couples to the proton coordinate. $[12-14]$

Recent works on the PCET theory have focused on the importance of the proton donoracceptor motion. The main concept is that fluctuations of the donor-acceptor separation $R_{D A}$ strongly affect the vibrational wave function overlap for the proton coordinate, and thus the effective transfer integral between vibronic states. Such effect has been first investigated in the case of vibrationally nonadiabatic proton transfer reactions, and is found to play an important role and leads to much smaller kinetic isotope effect (KIE) than simple estimation using the overlap of vibrational wave functions at the equilibrium proton donor-acceptor distance. [15 18] The effect of the donor-acceptor motion has also been widely discussed in recent PCET theories.[19-25] In these theoretical treatments, the proton degree of freedom is first quantized, and a Born-Oppenheimer approximation is applied to separate the proton motion and the slower degrees of freedom that couple to it. The PCET problem is then reduced to transitions between a group of vibronic states. Within the above theoretical framework, the effect of donor-acceptor motion can be treated either statically by doing a thermal average over the $R_{D A}$ distribution, [19, 23, 24] or can be treated dynamically using a time correlation function formalism. [20 22, 25]

It is interesting to investigate the applicability of the $\mathrm{BO}$ approximation to the proton motion for several reasons. First, although the masses of proton and deuterium are much less than those of the heavy atoms, the difference is not as dramatic as the mass ratio between the electron and nuclei. When the hydrogen bond is stiff between the donor and acceptor, high frequency donor-acceptor motion raise the question whether a separation of 
time scales is still appropriate. Second, even after the BO approximation is applied to separate the proton motion and the proton donor-acceptor motion, additional approximations such as static donor-acceptor motion, [19, 23, 24] or analytical approximations on the vibrational wave function overlap are often employed.[20, 22, 25] It is also desirable to test these approximations quantitatively using a solvable model.

In this paper, we provide tests of the above mentioned BO approximation for the proton motion using a model Hamiltonian for concerted PCET reactions. We show that, under certain well defined approximations, the effect of proton donor-acceptor motion can be incorporated in a model Hamiltonian base on the Duschinsky rotation effect (DRE).[26] The DRE describes the mixing of the normal modes between the donor and acceptor potential energy surfaces, [26] which is different from the linear displaced harmonic modes used in the conventional spin-boson model.[27, 28] During the past years, the effect of DRE have been widely discussed in areas such as electronic spectroscopy, 229 32] nonadiabatic relaxations, [29, 33, 34] and electron transfer reactions. [35-39] However, to the best of our knowledge, a model based on the DRE has not been applied in studies of PCET previously.

When the electronic coupling is small, the DRE model allows us to calculate the PCET reaction rates analytically using the Fermi's golden rule. This analytically solvable model is then applied to assess the BO approximation for the proton motion, as well as the static donor-acceptor motion approximation and the analytical approximations to the vibrational wave function overlaps. The remainder of this paper is arranged as follows. The model Hamiltonian and theories to calculate PCET rate constants are presented in Sec. II. The results are presented in Sec. III, where PCET rates and the KIEs are calculated using the FGR, and compared with results obtained from approximate theories. We have also studied the rate constant and KIE in the case of small electronic reorganization energy happen, [25, 40 42] with the focus on the possible role of vibrationally coherent tunneling. [43, 44] The main conclusions are summarized in Sec. IV. 


\section{THEORY}

\section{A. Model Hamiltonian}

We consider the case of concerted proton and electron transfer with a small electronic coupling (nonadiabatic ET), where a two electronic surfaces description is sufficient, [14, 22] and the Fermi's golden rule (FGR) that treats the electronic coupling to first order perturbation is valid. [28] To this end, we apply an extended spin-boson model to describe the PCET reactions. The total Hamiltonian can be written as

$$
H=H_{S}+H_{B}+H_{B S}
$$

where, the system Hamiltonian $H_{S}$ includes terms related to the electronic degree of freedom (DOF), the proton reaction coordinate $x$, and the proton donor-acceptor separation $R_{D A}$,

$$
H_{S}=\hbar \Delta \sigma_{x}+\frac{\Delta G}{2} \sigma_{z}+H_{a}|a\rangle\left\langle a\left|+H_{b}\right| b\right\rangle\langle b|
$$

where $|a\rangle$ and $|b\rangle$ denote the electron donor and acceptor states, $\sigma_{x}=|a\rangle\langle b|+| b\rangle\langle a|$, and $\sigma_{z}=|b\rangle\langle b|-| a\rangle\langle a| . H_{a}$ and $H_{b}$ are defined as

$$
H_{a, b}=\frac{p^{2}}{2 m_{H}}+\frac{P^{2}}{2 M}+\frac{1}{2} m_{H} \omega_{H}^{2}\left(x \pm \frac{d}{2} \pm \kappa \frac{R}{2}\right)^{2}+\frac{1}{2} M \Omega^{2} R^{2} .
$$

Here, $p$ and $x, P$ and $R$ are the momenta and coordinates of the proton and proton donoracceptor DOFs, respectively; $m_{H}$ and $\omega_{H}, M$ and $\Omega$ are the corresponding mass and frequencies; $R$ is defined as the difference between the donor-acceptor separation and its equilibrium value $R \equiv R_{D A}-R_{D A}^{e q} ; \kappa$ is the coupling coefficient between the proton equilibrium position and the proton donor-acceptor separation $R$, and $\pm(d+\kappa R) / 2$ is the equilibrium position of the proton coordinate on the donor and acceptor surfaces.

The potential energy surface presented in Eq. (3) leads to shifted proton equilibrium positions on the donor and acceptor surfaces as a function of the proton donor-acceptor distance $R$. As the $x$ and $R$ motions are coupled differently on the $|a\rangle$ and $|b\rangle$ surfaces, there is a rotation of the corresponding normal modes (see also Fig. 1), which is the DRE introduced in the previous Sec. I. In the case of a widely used linear model for the proton and the electron/proton donors and acceptors, [19 21, 25, 45] $\kappa=1$. Without loss of generality, we will assume such case and drop $\kappa$ in the following derivations. 
We now briefly discuss the relevance of the DRE in PCET reactions. The V-shaped $x-R$ potential energy surfaces for the donor and acceptor states as shown in Fig. 1 can actually be found in many previous publications studying proton transfer (PT) (e.g., Fig. 1 in Ref.[46]) and PCET reactions (e.g., Fig. 4 in Ref.[47]), as an effect that the proton equilibrium positions shift in different direction on the donor and acceptor surfaces as a function of the proton donor-acceptor distance. Under the harmonic approximation, these potential energy surfaces display a rotation of the normal modes that can be described by the DRE. The parameters for a DRE model can thus be obtained by analyzing the donor and acceptor potential energy surfaces.

We also assume that the solvent DOFs couple only to the electronic DOF, and the bath Hamiltonian can be written as

$$
H_{B}=\sum_{j=1}^{N}\left(\frac{p_{j}^{2}}{2 m_{j}}+\frac{1}{2} m_{j} \omega_{j}^{2} x_{j}^{2}\right),
$$

where $x_{j}, p_{j}, m_{j}, \omega_{j}$ are the coordinates, momenta, masses, and frequencies of the harmonic bath modes.

The bath DOFs are assumed to couple linearly with the electronic DOF, and the systembath coupling term is given by

$$
H_{B S}=-\sum_{j=1}^{N} c_{j} x_{j} \sigma_{z}
$$

The system-bath coupling is usually characterized using the spectral density $J(\omega)$ defined as

$$
J(\omega)=\frac{\pi}{2} \sum_{j=1}^{N} \frac{c_{j}^{2}}{m_{j} \omega_{j}} \delta\left(\omega-\omega_{j}\right) .
$$

In general, the proton DOF also couples to the environmental DOFs, which leads to a reorganization energy associated with proton motion, [12 14] as well as vibrational energy relaxation and dephasing. [48, 49] Although we did not consider such coupling in this paper, it can certainly be incorporated by extending the above model Hamiltonian described in Eqs. (1, 5). 


\section{B. Fermi's golden rule}

In the electronic nonadibatic limit where $\hbar \Delta$ is small, first order perturbation can be applied, and the rate constant can be calculated using Fermi's Golden Rule:[28]

$$
k=\Delta^{2} \int_{-\infty}^{\infty} e^{-i \frac{\Delta G}{\hbar} t} C(t) d t
$$

where the correlation function $C(t)$ is defined as

$$
C(t)=\frac{1}{Z_{0}} \operatorname{Tr}\left[e^{-\beta H_{0}} e^{\frac{i}{\hbar} H_{0} t} e^{-\frac{i}{\hbar} H_{1} t}\right] .
$$

Here, $Z_{0}$ is the partition function of the donor state, $Z_{0}=\operatorname{Tr} e^{-\beta H_{0}}$,

$$
H_{0}=H_{a}+H_{B}+\sum_{j} c_{j} x_{j}
$$

and

$$
H_{1}=H_{b}+H_{B}-\sum_{j} c_{j} x_{j}
$$

Since the proton and proton donor-acceptor DOFs are decoupled from the bath modes, $C(t)$ can be calculated as

$$
C(t)=\frac{1}{Z_{a}} \operatorname{Tr}\left[e^{-\beta H_{a}} e^{\frac{i}{\hbar} H_{a} t} e^{-\frac{i}{\hbar} H_{b} t}\right] C_{B}(t) .
$$

$C_{B}(t)$ on the right hand side of the above Eq. (11) arises from the bath contribution and is defined as

$$
C_{B}(t)=\frac{1}{\operatorname{Tr} e^{-\beta H_{B 0}}} \operatorname{Tr}\left[e^{-\beta H_{B 0}} e^{\frac{i}{\hbar} H_{B 0} t} e^{-\frac{i}{\hbar} H_{B 1} t}\right]
$$

where $H_{B 0, B 1}=H_{B} \pm \sum_{j} c_{j} x_{j}$. It can be calculated analytically using the spectral density $J(\omega)$, resulting in the following equation:[28]

$$
C_{B}(t)=\exp \left\{-\frac{4}{\hbar} \int_{0}^{\infty} d \omega \frac{J(\omega)}{\pi \omega^{2}}[\operatorname{coth}(\beta \hbar \omega / 2)(1-\cos \omega t)+i \sin \omega t]\right\} .
$$

As in the many previous theories for the PCET reactions, we apply the following approximation for $C_{B}(t)$, which can usually be obtained by a high temperature approximation and short-time expansion:

$$
C_{B}(t) \approx e^{-\frac{\lambda t^{2}}{\beta \hbar^{2}}-i \frac{\lambda}{\hbar} t}
$$

where $\lambda$ is the electronic reorganization energy

$$
\lambda=\int_{0}^{\infty} d \omega \frac{4 J(\omega)}{\pi \omega} .
$$


The first term on the right hand side of the above Eq. (11), $\frac{1}{Z_{a}} \operatorname{Tr}\left[e^{-\beta H_{a}} e^{\frac{i}{\hbar} H_{a} t} e^{-\frac{i}{\hbar} H_{b} t}\right]$ describes the time correlation function of a two-mode DRE model, and can be calculated as

$$
\frac{1}{Z_{a}} \operatorname{Tr}\left[e^{-\beta H_{a}} e^{\frac{i}{\hbar} H_{a} t} e^{-\frac{i}{\hbar} H_{b} t}\right]=\frac{1}{Z_{a}} \int d x d R d x^{\prime} d R^{\prime}\left\langle x R\left|e^{\frac{i}{\hbar} H_{a}(t+i \hbar \beta)}\right| x^{\prime} R^{\prime}\right\rangle\left\langle x^{\prime} R^{\prime}\left|e^{-\frac{i}{\hbar} H_{b} t}\right| x R\right\rangle .
$$

Since $H_{a}$ and $H_{b}$ are Hamiltonians of harmonic oscillators, the matrix elements can be calculated analytically, [50] and the correlation function can then be obtained using Gaussian integrals. Details of such calculation can be found in many previous publications such as Refs. [29, 31, 34, 39], and will not be presented in this paper.

We further note that the harmonic $x$ - $R$ model was employed in this study in order to obtain an analytical expression for the time correlation function in Eq. (11). Including the anharmonic effects numerically in some of the widely used linear models (e.g., Refs. [21, 40, 41]) would also be straightforward, as calculating Eq. (16) in such cases only need to solve a two-dimensional Schrödinger equation, which can be done routinely on modern computers. We note that a similar idea of solving a two-dimensional Schrödinger equation for the proton and proton accepter-donor motion was proposed in Ref.[20].

\section{Rate constant within the BO approximation for the proton motion}

Due to the small mass of proton or deuterium, a BO approximation is often applied to separate the motion of the proton coordinates $x$ and the proton donor-acceptor separation $R$. For a fixed donor-acceptor separation $R$, the proton coordinate can be quantized, and the vibronic states can be calculated as

$$
\left[\frac{p^{2}}{2 m_{H}}+V_{\alpha}(x, R)\right]\left|\phi_{j, \alpha}(x, R)\right\rangle=E_{j, \alpha}\left|\phi_{j, \alpha}(x, R)\right\rangle,
$$

where $\alpha=a, b$ denotes the electronic donor and acceptor states, and $j$ denotes the vibrational states for the proton DOF. According to Eq. (3),$V_{a, b}(x, R)=1 / 2 m_{H} \omega_{H}^{2}(x \pm d / 2 \pm R / 2)^{2}$, and $E_{j, \alpha}$ and $\left|\phi_{j, \alpha}(x, R)\right\rangle$ can be obtained from the eigenstates of displaced harmonic-oscillators.

In the case of small electronic coupling considered in the above subsection, neglecting the non-BO coupling terms between the vibronic states in Eq. (17) will formulate the PCET reaction as nonadiabtic transitions between a group of vibronic states. [6, 요, 14, 22] By further assuming an initial equilibrium distribution on the donor vibrational states, the total rate 
constant can be calculated as

$$
k=\sum_{\mu \nu} P_{\mu, a} k_{\mu \nu}
$$

where $P_{\mu, a}$ is the equilibrium population of vibrational state $|\mu\rangle$ on the donor potential energy surface; and $k_{\mu \nu}$ is the transition rate constant from state $|\mu, a\rangle$ to $|\nu, b\rangle$. The rate constant $k_{\mu \nu}$ are then calculated using the effective system Hamiltonian involving states $|\mu, a\rangle$ and $|\nu, b\rangle[\underline{6}, \underline{8}, \underline{14}, \underline{22}]$

$$
\begin{aligned}
H_{S}^{\mu \nu}= & \hbar \Delta\left\langle\phi_{\mu} \mid \phi_{\nu}\right\rangle(|\mu, a\rangle\langle\nu, b|+c . c .)+\left[-\Delta G / 2+(\mu+1 / 2) \hbar \omega_{H}\right]|\mu, a\rangle\langle\mu, a| \\
& {\left[\Delta G / 2+(\nu+1 / 2) \hbar \omega_{H}\right]|\nu, b\rangle\langle\nu, b|+\frac{P^{2}}{2 M}+\frac{1}{2} M \Omega^{2} R^{2} . }
\end{aligned}
$$

Since fluctuations of the the donor-acceptor coordinate $R$ can cause changes of the overlap integral $\left\langle\phi_{\mu} \mid \phi_{\nu}\right\rangle$ by orders of magnitudes, [15-20, 22, 23, 25] there is no general exact analytical expression for $k_{\mu \nu}$, and additional approximations are often employed [6, 8, 14, 18, 22, 51]. We will briefly present the results in applying two widely used approximations to the model system presented in the previous subsections II. A and B in the case of nonadiabatic ET reactions.

In the rate constant expression originally proposed by Kutnetsov and Ulstrup, [51] the $R$-mode is treated statically, and the rate constant is obtained as a thermally average over the classical Boltzmann distribution of $R$ :

$$
k_{\mu \nu} \approx \Delta^{2} \sqrt{\frac{\pi}{\lambda k_{B} T}} \exp \left[-\frac{\left(\Delta G_{\mu \nu}+\lambda\right)^{2}}{4 \lambda k_{B} T}\right] \int d R P(R)\left|S_{\mu \nu}(R)\right|^{2},
$$

where $\Delta G_{\mu \nu}=\Delta G+(\nu-\mu) \hbar \omega_{H}, S_{\mu \nu}(R)=\left\langle\phi_{\mu}(R) \mid \phi_{\nu}(R)\right\rangle$ is the Franck-Condon overlap of the vibrational wave functions, and $P(R)=\sqrt{M \Omega^{2} / 2 \pi k_{B} T} e^{-M \Omega^{2} R^{2} / 2 k_{B} T}$. This static approximation has recently been applied to PCET reactions in enzymes by Klinman and coworkers.[19, 23] For cases where $\hbar \Omega>k_{B} T$, the above Eq. (20) can be extended to include the quantum effect of the $R$-mode by using a quantum mechanic distribution for the $R$-mode, [18, 52]

$$
k_{\mu \nu} \approx \Delta^{2} \sqrt{\frac{\pi}{\lambda k_{B} T}} \exp \left[-\frac{\left(\Delta G_{\mu \nu}+\lambda\right)^{2}}{4 \lambda k_{B} T}\right] \int d R P_{q m}(R)\left|S_{\mu \nu}(R)\right|^{2},
$$

where $P_{q m}(R)=\sqrt{1 / 2 \pi\left\langle R^{2}\right\rangle} e^{-R^{2} / 2\left\langle R^{2}\right\rangle}$, and $\left\langle R^{2}\right\rangle=\hbar / 2 M \Omega \operatorname{coth}(\beta \hbar \Omega / 2)$. This extended UK expression will be used in later calculations for the static $R$-mode approximation. 
Another approximation is to expand $S_{\mu \nu}(R)$ around the equilibrium position $R=0$ using an exponential function, which is widely used in PT[17, 53, 54] and PCET[20, 22] theories:

$$
S_{\mu \nu}(R) \approx S_{\mu \nu}(0) e^{-\alpha_{\mu \nu} R}
$$

The problem is now equivalent to an extended spin-boson model with exponential coupling. [55] $k_{\mu \nu}$ can then be calculated as [20, 22]

$$
k_{\mu \nu}=\left|\Delta S_{\mu \nu}(0)\right|^{2} \int_{-\infty}^{\infty} d t \exp \left[\alpha_{\mu \nu}^{2}\left(C_{R}(0)+C_{R}(t)\right)\right] e^{-i \frac{\Delta G \mu \nu}{\hbar} t-\frac{\lambda t^{2}}{\beta \hbar^{2}}-i \frac{\lambda}{\hbar} t}
$$

where

$$
C_{R}(t)=\frac{\hbar}{2 M \Omega}[\operatorname{coth}(\beta \hbar \Omega / 2) \cos \Omega t-i \sin \Omega t] .
$$

In later studies, we will denote Eqs. (18), (23), and (24), as the exponential coupling approximation.

\section{RESULTS}

\section{A. Rate constant and KIE}

We now apply the above theories to calculate PCET rate constants and KIEs. Fig. 1] shows the donor and acceptor potential energy surfaces from the Hamiltonian $H_{a}$ and $H_{b}$ defined in Eq. (3). We will assume $\omega_{H}=3000 \mathrm{~cm}^{-1}$ throughout this paper, the other parameters used in Fig. 1 are $M=100 \mathrm{amu}, \Omega=100 \mathrm{~cm}^{-1}$, and $d=0.45 \AA$. It can be seen that when $R$ is smaller than its equilibrium value $R=0$, the distance between the energy minima for the proton coordinate on the donor and acceptor surfaces becomes smaller, which will lead to enhanced proton tunnelling. A key feature of the DRE, which is the rotation between normal modes on the donor and acceptor potential energy surfaces, can be clearly seen in Fig. 1.

Fig. 2 shows the PCET rate constant as a function of the driving force $\Delta G$ using the DRE model, where the proton coordinate $x$ is coupled to $R$-modes with different parameters. $\lambda=$ $30 \mathrm{kcal} / \mathrm{mol}, \Delta=100 \mathrm{~cm}^{-1}, d=0.45 \AA$, and $T=300 \mathrm{~K}$ were used in the calculation. It can be seen that coupling to the $R$-mode can significantly increase the rate constants by orders of magnitude, especially when the donor-acceptor mass is small. We also note that the PCET rate constants keep increasing with more negative $\Delta G<-\lambda$. The reason is that the overall 
reorganization energy is very large after taking account into the contribution from the proton coordinate, and a real turnover of the PCET rate can only happen at very negative $\Delta G$ (see the inset of Fig. (2)). This effect was also observed in recent studies by Hammes-Schiffer and coworkers, [41, 56] where the explanation is based on vibronic transitions to high vibrational states. Such high activation barrier for ET indicates that the proton transfer should happen mainly through quantum tunnelling.

The KIE is an important character of reactions involving proton transfer, which is defined as the ratio of the rate constant for hydrogen to that for deuterium. The dependence of PCET rate constants and KIEs on various parameters has been widely discussed in previous theoretical studies. 86, 14, 22 24] More specifically, the effects of different donor-acceptor parameters and driving forces have been investigated by Hammes-Schiffer and coworkers recently. [41, 56] The main purpose of the calculations below is, however, to quantitatively assess the applicability of the $\mathrm{BO}$ approximation for the proton motion: the static $R$-mode and the exponential coupling approximations will be evaluated using the analytically solvable model Hamiltonian presented in Sec. II.

Fig. 3 plots the PCET reaction rate constants for proton and deuterium as a function of the donor-acceptor motion frequency. Comparisons with the rate constant expression using exponential coupling approximation (Eqs. 18, 23, and 24), and the extended UK expression within the static $R$-mode approximation (Eqs. 18 and 21) are also shown. Three different donor-acceptor masses $M=100 \mathrm{amu}, 20 \mathrm{amu}$, and $7 \mathrm{amu}$ were considered. The other parameters are $\lambda=30 \mathrm{kcal} / \mathrm{mol}, \Delta G=-5 \mathrm{kcal} / \mathrm{mol}, d=0.45 \AA$, and $T=300 \mathrm{~K}$. It can be seen that the rate constant increases significantly with the decrease of the $R$-mode mass, where larger proton donor-acceptor fluctuation leads to larger enhancement of the PCET rates. The extended UK rate expression Eq. (21) agrees well with the exact FGR result except for the small mass and high frequency cases $\left(M=20 \mathrm{amu}\right.$ and $\Omega>500 \mathrm{~cm}^{-1}$, $M=7$ amu and $\Omega>400 \mathrm{~cm}^{-1}$ ), which indicates that the dynamical effect of the $R$-mode becomes important only at high frequencies. We can also see that the exponential coupling approximation Eq. (23) starts to overestimate the rate constants and becomes inaccurate for small mass and small donor-acceptor frequency $\left(\Omega<200 \mathrm{~cm}^{-1}\right.$ for $M=100 \mathrm{amu}, \Omega<400$ $\mathrm{cm}^{-1}$ for $M=20 \mathrm{amu}, \Omega<700 \mathrm{~cm}^{-1}$ for $M=7 \mathrm{amu}$ ), with a steep rise of the rate constants for low donor-acceptor frequency $\Omega$. The reason is that, the range of fluctuation for $R$ is quite large in such cases, and the exponential approximation for the vibrational wave 
function overlap in Eq. (22) becomes invalid.

Fig. 4 plots the KIE as a function of the donor-acceptor frequency calculated with different rate expressions. It can be seen that the KIEs are small at low frequencies, which means that the enhancement of the PCET rate due to $R$-mode fluctuations is more significant for deuterium. The extended UK method agrees well with the FGR result except for the case of high proton donor-acceptor frequencies $\left(\Omega>500 \mathrm{~cm}^{-1}\right.$ for $M=20 \mathrm{amu}$, and $\Omega>400$ $\mathrm{cm}^{-1}$ for $M=7 \mathrm{amu}$.). The rate constant expression Eq. (23) has problems in cases of small mass and frequency. This problem is more severe when the donor-acceptor mass $M$ is small (e.g., the left parts of Fig. 4(b) and (c)) as the exponential approximation in Eq. (22) becomes inaccurate when the fluctuation of $R$ is large. We note that the failure of the exponential overlap approximation in calculating KIEs at low donor-acceptor frequencies has been discussed recently, [41] while it is more quantitatively characterized in Fig,4 in this study by comparing to the exact FGR result.

In summarizing this subsection, we have shown that the model Hamiltonian including the DRE captures the main features of PCET reactions presented in many previous theoretical studies. [8, 14, 22 24] The advantage of the current model is that, it can include the effect of the proton donor-acceptor motion without the BO approximation for the proton motion, as well as further approximations for the vibrational wave function overlap. Our quantitative evaluation of the static $R$-mode and the exponential overlap approximations could also be helpful in developing more accurate PCET theory in more general cases.

\section{B. Vibrational coherence at small reorganization energy}

Recently, abnormal temperature dependence of KIE were observed in experimental stud-

ies of several systems, [57, 58] where the KIE increases as the temperature increases, implying a larger apparent activation free energy for proton transfer. Theoretical studies has pointed out that this could be a phenomena associated with low reorganization energies. [25, 41, 42] An interesting finding in the literature is that, when the reorganization energy is small (i.e., in the low friction regime), coherent tunneling may play a role in the ET dynamics. [43, 44] It is thus interesting to investigate whether coherent tunneling is relevant to the abnormal temperature dependence of KIE in the case of small reorganization energies.

We plot in Fig. 5 the dependence of the PCET rate constant as a function of the driving 
force $\Delta G$ for $\lambda=3 \mathrm{kcal} / \mathrm{mol}$. The other parameters are $M=20 \mathrm{amu}, \Omega=300 \mathrm{~cm}^{-1}$, and $d=0.45 \AA$. The rate constants show oscillations as a function of $\Delta G$, and this oscillatory behavior is more pronounced for proton than deuterium. This effect is readily explained in the picture of the BO approximation, [41, 42, 56] with the most recent explanation presented in Ref.[56]. For example, using the extended UK type of expressions: as the vibrational energy levels for proton motion is $3000 \mathrm{~cm}^{-1}$, or approximately $8.6 \mathrm{kcal} / \mathrm{mol}$, the small reorganization energy $\lambda=3 \mathrm{kcal} / \mathrm{mol}$ is not large enough to bridge the gaps between each pairs of vibronic transitions, and peaks for rate constants occur at approximately $\Delta G_{\mu \nu}+\lambda \approx$ 0. As the deuterium case has a smaller vibrational spacing, this also explains the less oscillatory behavior for deuterium. Based on the same reasoning, Hammes-Schiffer and coworkers have also explained the abnormal temperature dependence of the KIE in the case of small $\lambda .[41,42]$

Here we provide a complementary view to the above explanation that was based on the BO approximation to the proton motion. In the FGR rate expression Eq. (17), the real part of the integrand $C_{\Delta G}(t) \equiv \operatorname{Re}\left[e^{-i \frac{\Delta G}{\hbar} t} C(t)\right]$ can be regarded as the (normalized) reaction flux correlation function whose integral gives the rate constant. Within first order approximation, the time dependent acceptor population at short times can also be estimated as

$$
\frac{d}{d t} P_{a}(t) \approx k(t) P_{d}(0)
$$

where $P_{a}$ is the acceptor population, $P_{d}$ is the donor population, and

$$
k(t)=2 \Delta^{2} \operatorname{Re} \int_{0}^{t} d t C_{\Delta G}(t) .
$$

The normalized flux correlation function $C_{\Delta G}(t)$ is plotted in Fig. 6 (a), for two different $\Delta G$ values at $-3.5 \mathrm{kcal} / \mathrm{mol}$ and $-6.5 \mathrm{kcal} / \mathrm{mol}$. The corresponding population evolution is shown in Fig. 6 (b) by assuming $P_{d}(0)=1$. We can see that due to the smaller damping effect associated with small $\lambda$, the second and third peaks in the flux correlation function starts to contribute to the rate constant, and their contribution can be either constructive (for $\Delta G=-3.5 \mathrm{kcal} / \mathrm{mol}$, the contribution from second peak is $40 \%$ from the first peak) or destructive, (for $\Delta G=-6.5 \mathrm{kcal} / \mathrm{mol}$, the contribution from second peak is $-70 \%$ from the first peak), which leads to peaks or valleys in the rate constant as shown in Fig. 5, We note that this just the vibrationally coherent tunneling effect observed in the previous numerical studies of photo-induced electron transfer reaction involving a high frequency intramolecular mode, [44] although a slightly different model is used in the studies of Ref.[44]. 
An interesting observation here is that, at $\Delta G$ values near the peaks and valleys of the rate constant curve in Fig. 5, the temperature dependence of the KIE shows different behavior. Fig. 7 plots the KIE as a function of temperature for $\Delta G=-3.5 \mathrm{kcal} / \mathrm{mol}$ (close to a peak), which decreases when the temperature increases; while for $\Delta G=-6.5 \mathrm{kcal} / \mathrm{mol}$ (close to a valley), the KIE shows abnormal behavior as it increases with increasing temperature. The dashed lines shows the results by including only the first peak contribution from $C_{\Delta G}(t)$, which shows the normal temperature dependence in both case. Comparing the solid and dashed curves thus shows that the contribution from the oscillations in $C_{\Delta G}(t)$ play an important role in the abnormal temperature dependence for $\Delta G=-6.5 \mathrm{kcal} / \mathrm{mol}$. This observation also holds for $\Delta G$ values near other peaks and valleys. So we conclude that the abnormal temperature dependence of KIE in the case of small reorganization energy is related to coherent vibrational tunneling, which is a new explanation complimentary to the previous ones within the $\mathrm{BO}$ approximation of the proton motion.

\section{CONCLUSIONS}

Using a model Hamiltonian based on the Duschinsky rotation effect, we were able to quantitatively investigate the applicability of the commonly used BO approximation for the proton motion in studying PCET reactions. The extended UK method using the static $R$ mode approximation was found to work reasonably well except for small donor-acceptor mass and high donor-acceptor frequencies. The exponential approximation for the vibrational function overlap was also tested in the model systems, and found to be inaccurate in cases of large proton-donor separation fluctuations when the proton donor-acceptor mass and frequency are small. This may suggest that more accurate analytical approximations are needed in such cases.

In the case of small reorganization energies, we find that coherent vibrational tunneling can lead to oscillations of rate constants with respect to the driving force $\Delta G$, and different temperature dependence of KIE at $\Delta G$ near the peaks and valleys on the rate-driving force curve in Fig. 5, Our explanation of these results based on coherent tunnelling is complementary to existing theories within the BO approximation for proton motion. [25, 41, 42, 56] As the vibrational coherent tunnelling is sensitive to couplings of the proton coordinate

to the environmental DOFs, [44] extending the current study to incorporate such couplings 
would certainly be interesting. The current work could also be extended to investigate photo-induced PCET reactions, [59, 60] where non-equilibrium dynamics involving vibrational motion is important.

\section{Acknowledgments}

This work is supported by NNSF of China (20733006, 20873157, 20903101), and the Chinese Academy of Sciences (KJCX2.YW.H17 and the Hundred Talents Project).

[1] J. M. Mayer, Annu. Rev. Phys. Chem. 55, 363 (2004).

[2] C. Tommos and G. T. Babcock, Acc. Chem. Res. 31, 18 (1998).

[3] C. Costentin, M. Robert, and J. M. Saveant, J. Am. Chem. Soc. 128, 4552 (2006).

[4] M. H. V. Huynh and T. J. Meyer, Chem. Rev. 107, 5004 (2007).

[5] S. Y. Reece and D. G. Nocera, Annu. Rev. Biochem. 78, 673 (2009).

[6] R. I. Cukier, J. Phys. Chem. 98, 2377 (1994).

[7] R. I. Cukier, J. Phys. Chem. 100, 15428 (1996).

[8] R. I. Cukier and D. G. Nocera, Annu. Rev. Phys. Chem. 49, 337 (1998).

[9] J. Jortner, Mol. Phys. 32, 379 (1976).

[10] R. A. Marcus, Annu. Rev. Phys. Chem. 15, 155 (1964).

[11] R. A. Marcus and N. Sustin, Biochim. Biophys. Acta 811, 265 (1985).

[12] A. Soudackov and S. Hammes-Schiffer, J. Chem. Phys. 111, 4672 (1999).

[13] A. Soudackov and S. Hammes-Schiffer, J. Chem. Phys. 113, 2385 (2000).

[14] S. Hammes-Schiffer, Acc. Chem. Res. 34, 273 (2001).

[15] D. Borgis and J. T. Hynes, J. Chem. Phys. 94, 3619 (1991).

[16] A. Suárez and R. Silbey, J. Chem. Phys. 94, 4809 (1991).

[17] P. M. Kiefer and J. T. Hynes, J. Phys. Chem. A 108, 11793 (2004).

[18] P. M. Kiefer and J. T. Hynes, J. Phys. Chem. A 108, 11809 (2004).

[19] M. J. Knapp, K. Rickert, and J. P. Klinman, J. Am. Chem. Soc. 124, 3865 (2002).

[20] A. Soudackov, E. Hatcher, and S. Hammes-Schiffer, J. Chem. Phys. 122, 014505 (2005).

[21] E. Hatcher, A. V. Soudackov, and S. Hammes-Schiffer, Chem. Phys. 319, 93 (2005). 
[22] S. Hammes-Schiffer and A. V. Soudackov, J. Phys. Chem. B 112, 14108 (2008).

[23] J. P. Klinman, Biochim. Biophys. Acta 1757, 981 (2006).

[24] R. I. Cukier, Biochim. Biophys. Acta 1655, 37 (2004).

[25] S. Presse and R. J. Silbey, J. Chem. Phys. 124, 124504 (2006).

[26] F. Duschinsky, Acta Physicochim. URSS 7, 551 (1937).

[27] A. J. Leggett, S. Chakravarty, A. T. Dorsey, M. Fisher, A. Garg, and W. Zwerger, Rev. Mod. Phys. 59, 1 (1987).

[28] U. Weiss, Quantum dissipative systems (World Scientific, London, 1999), 2nd ed.

[29] R. Kubo and Y. Toyozawa, Prog. Theoret. Phys. 13, 160 (1955).

[30] Y. J. Yan and S. Mukamel, J. Chem. Phys. 85, 5908 (1986).

[31] S. A. Egorov, E. Rabani, and B. J. Berne, J. Chem. Phys. 108, 1407 (1998).

[32] A. M. Mebel, M. Hayashi, K. K. Liang, and S. H. Lin, J. Phys. Chem. A 103, 10674 (1999).

[33] S. A. Egorov, E. Rabani, and B. J. Berne, J. Chem. Phys. 110, 5238 (1999).

[34] Q. Peng, Y. Yi, Z. Shuai, and J. Shao, J. Chem. Phys. 126, 114302 (2007).

[35] E. J. Lee, E. S. Medvedev, and A. A. Stuchebrukhov, J. Chem. Phys. 112, 9015 (2000).

[36] G. M. Sando, K. G. Spears, J. T. Hupp, and P. T. Ruhoff, J. Phys. Chem. A 105, 5317 (2001).

[37] J. Tang, M. T. Lee, and S. H. Lin, J. Chem. Phys. 119, 7188 (2003).

[38] K. K. Liang, A. M. Mebel, S. H. Lin, M. Hayashi, H. L. Selzle, E. W. Schlag, and M. Tachiya, Phys. Chem. Chem. Phys. 5, 4656 (2003).

[39] K. A. Velizhanin and H. B. Wang, J. Chem. Phys. 131, 094109 (2009).

[40] E. Hatcher, A. V. Soudackov, and S. Hammes-Schiffer, J. Am. Chem. Soc. 129, 187 (2007).

[41] S. J. Edwards, A. V. Soudackov, and S. Hammes-Schiffer, J. Phys. Chem. A 113, 2117 (2009).

[42] M. K. Ludlow, A. V. Soudackov, and S. Hammes-Schiffer, J. Am. Chem. Soc. 131, 7094 (2009).

[43] J. N. Onuchic and P. G. Wolynes, J. Phys. Chem. 92, 6495 (1988).

[44] M. Topaler and N. Makri, J. Phys. Chem. 100, 4430 (1996).

[45] E. Hatcher, A. V. Soudackov, and S. Hammes-Schiffer, J. Phys. Chem. B 109, 18565 (2005).

[46] J. Pu, J. Gao, and D. G. Truhlar, Chem. Rev. 106, 3140 (2006).

[47] E. Hatcher, A. V. Soudackov, and S. Hammes-Schiffer, J. Am. Chem. Soc. 126, 5763 (2004).

[48] D. W. Oxtoby, Adv. Chem. Phys. 47, 487 (1981).

[49] J. C. Owrutsky, D. Raftery, and R. M. Hochstrasser, Annu. Rev. Phys. Chem. 45, 519 (1994). 
[50] R. P. Feynman and A. R. Hibbs, Quantum Mechanics and Path Integrals (McGraw-Hill, New York, 1965).

[51] A. Kuznetsov and J. Ulstrup, Can. J. Chem. 77, 1085 (1999).

[52] S. Hammes-Schiffer, E. Hatcher, H. Ishikita, J. H. Skone, and A. V. Soudackov, Coord. Chem. Rev. 252, 384 (2008).

[53] D. Borgis, S. Lee, and J. T. Hynes, Chem. Phys. Lett. 162, 19 (1989).

[54] A. Suárez and R. Silbey, J. Chem. Phys. 95, 9115 (1991).

[55] A. Nitzan, S. Mukamel, and J. Jortner, J. Chem. Phys. 63, 200 (1975).

[56] S. J. Edwards, A. V. Soudackov, and S. Hammes-Schiffer, J. Phys. Chem. B 113, 14545 (2009).

[57] J. L. Cape, M. K. Bowman, and D. M. Kramer, J. Am. Chem. Soc. 127, 4208 (2005).

[58] J. M. Hodgkiss, N. H. Damrauer, S. Presse, J. Rosenthal, and D. G. Nocera, J. Phys. Chem. B 110, 18853 (2006).

[59] B. Li, J. Zhao, K. Onda, K. D. Jordan, J. L. Yang, and H. Petek, Science 311, 1436 (2006).

[60] C. Venkataraman, A. V. Soudackov, and S. Hammes-Schiffer, J. Chem. Phys. 131, 154502 (2009). 


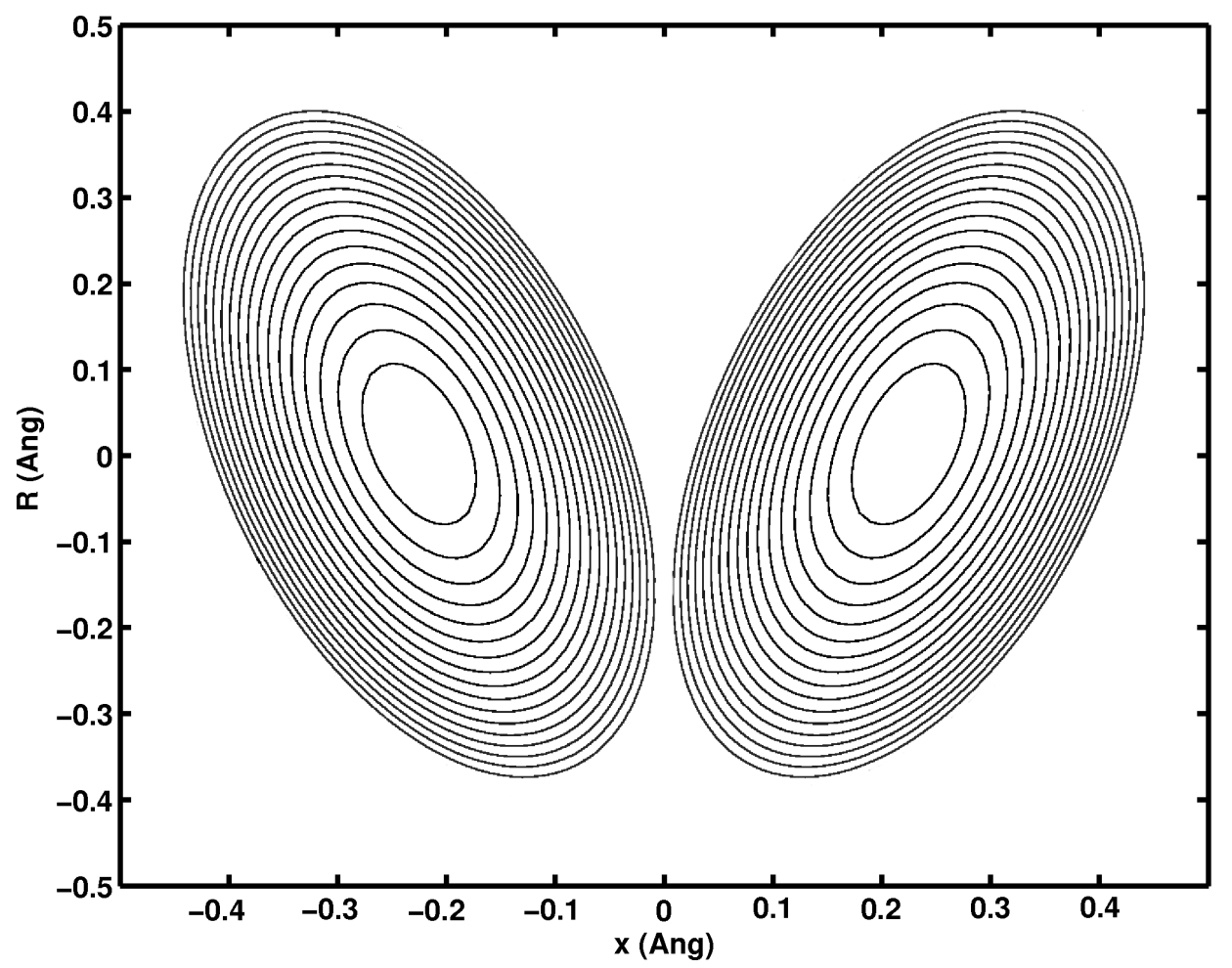

FIG. 1: Potential energy surfaces for the electronic donor (left) and acceptor (right) states. 


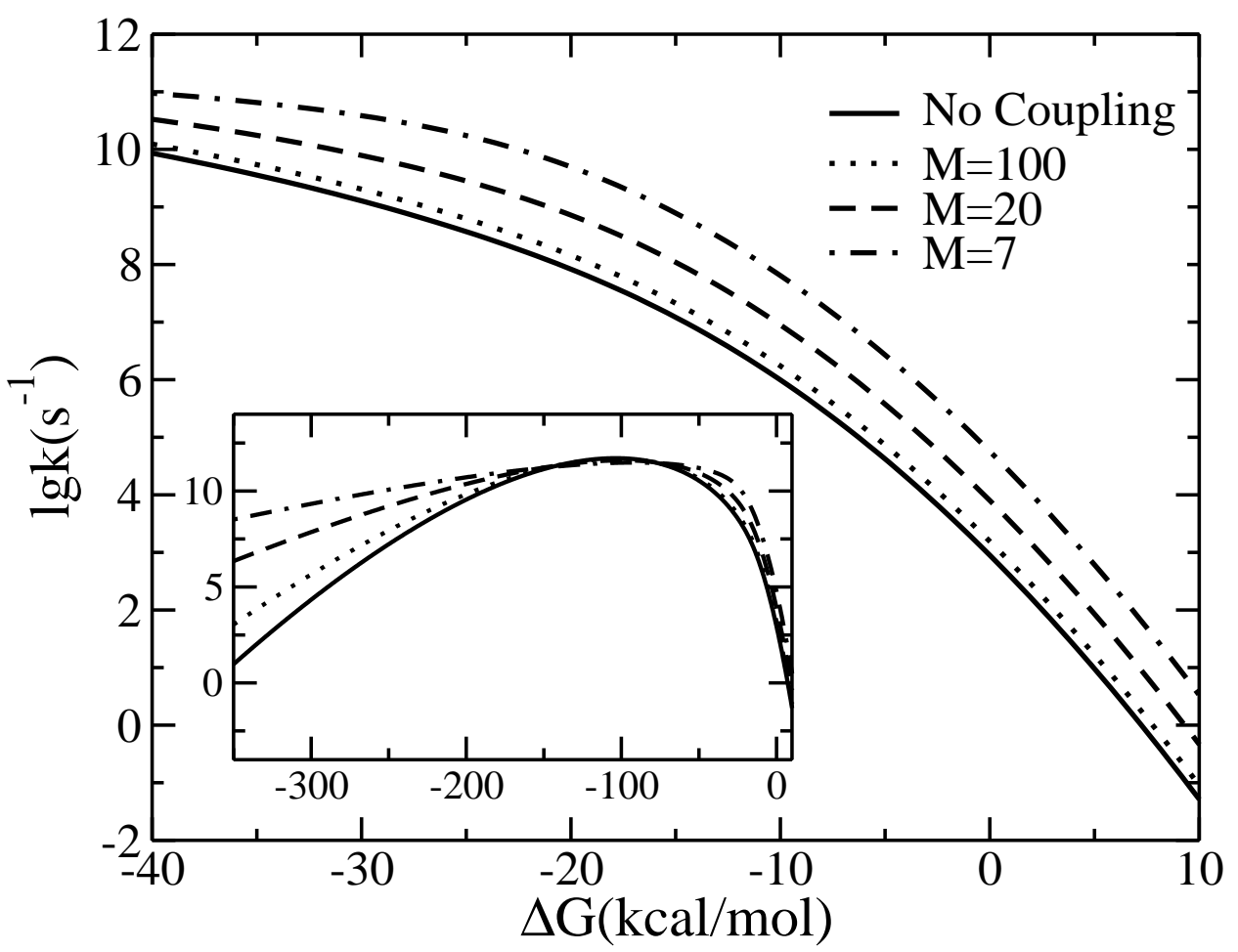

FIG. 2: PCET rate constants as a function of $\Delta G$. Curves from bottom to top: No coupling to the $R$-mode; $M=100 \mathrm{amu}, M=20 \mathrm{amu}$, and $M=7 \mathrm{amu}$. The other parameters are: $\lambda=30$ $\mathrm{kcal} / \mathrm{mol}, \Delta=100 \mathrm{~cm}^{-1}, \Omega=300 \mathrm{~cm}^{-1}, d=0.45 \AA$, and $T=300 \mathrm{~K}$. 


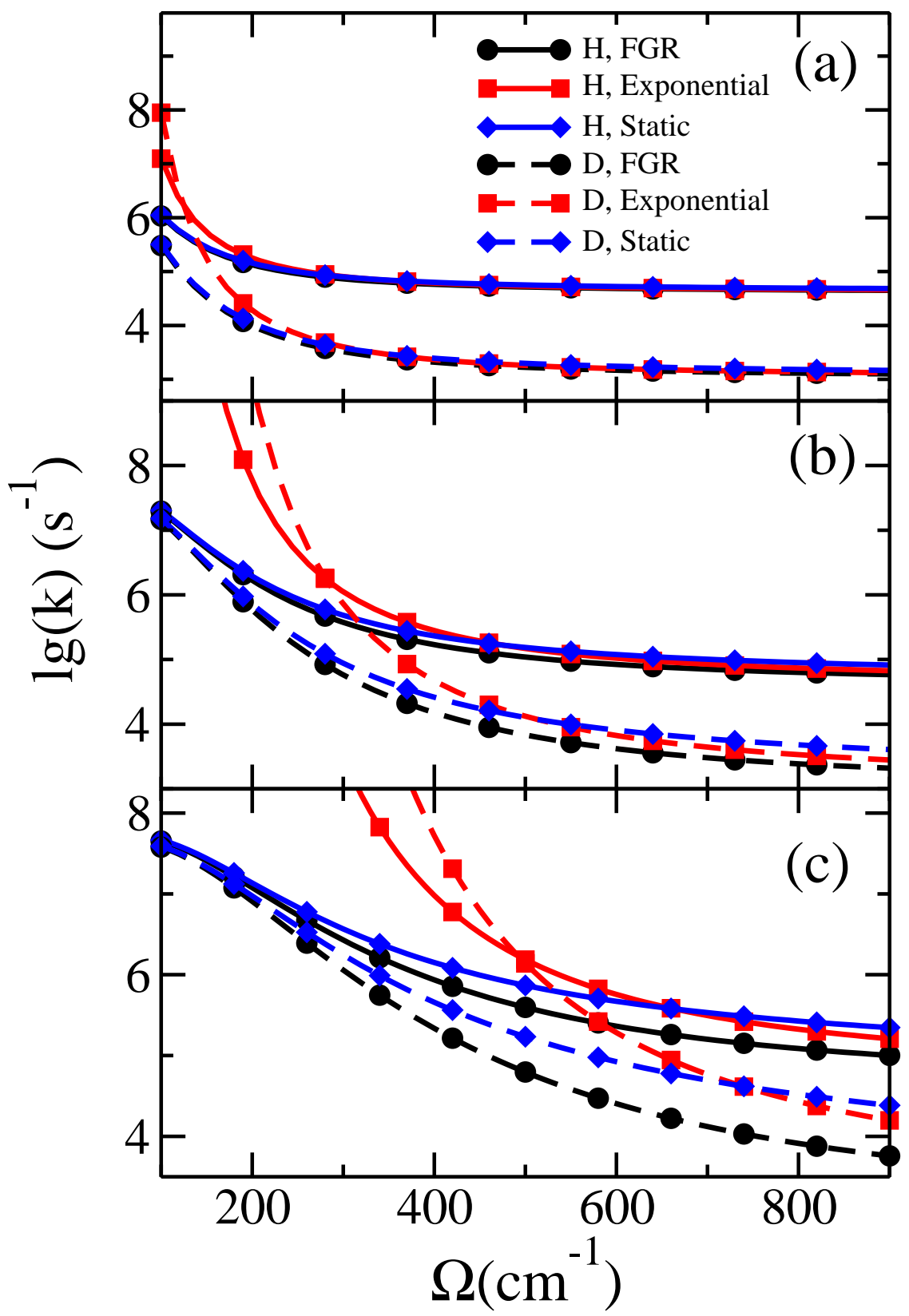

FIG. 3: Rate constants as a function of the proton donor-acceptor frequency $\Omega$ for proton (H) and deuterium (D). (a) $\mathrm{M}=100 \mathrm{amu}$, (b) $\mathrm{M}=20 \mathrm{amu}$, (c) $\mathrm{M}=7 \mathrm{amu}$. The other parameters are $\lambda$ $=30 \mathrm{kcal} / \mathrm{mol}, \Delta G=-5 \mathrm{kcal} / \mathrm{mol}, \Delta=100 \mathrm{~cm}^{-1}, d=0.45 \AA$, and $T=300 \mathrm{~K}$. 


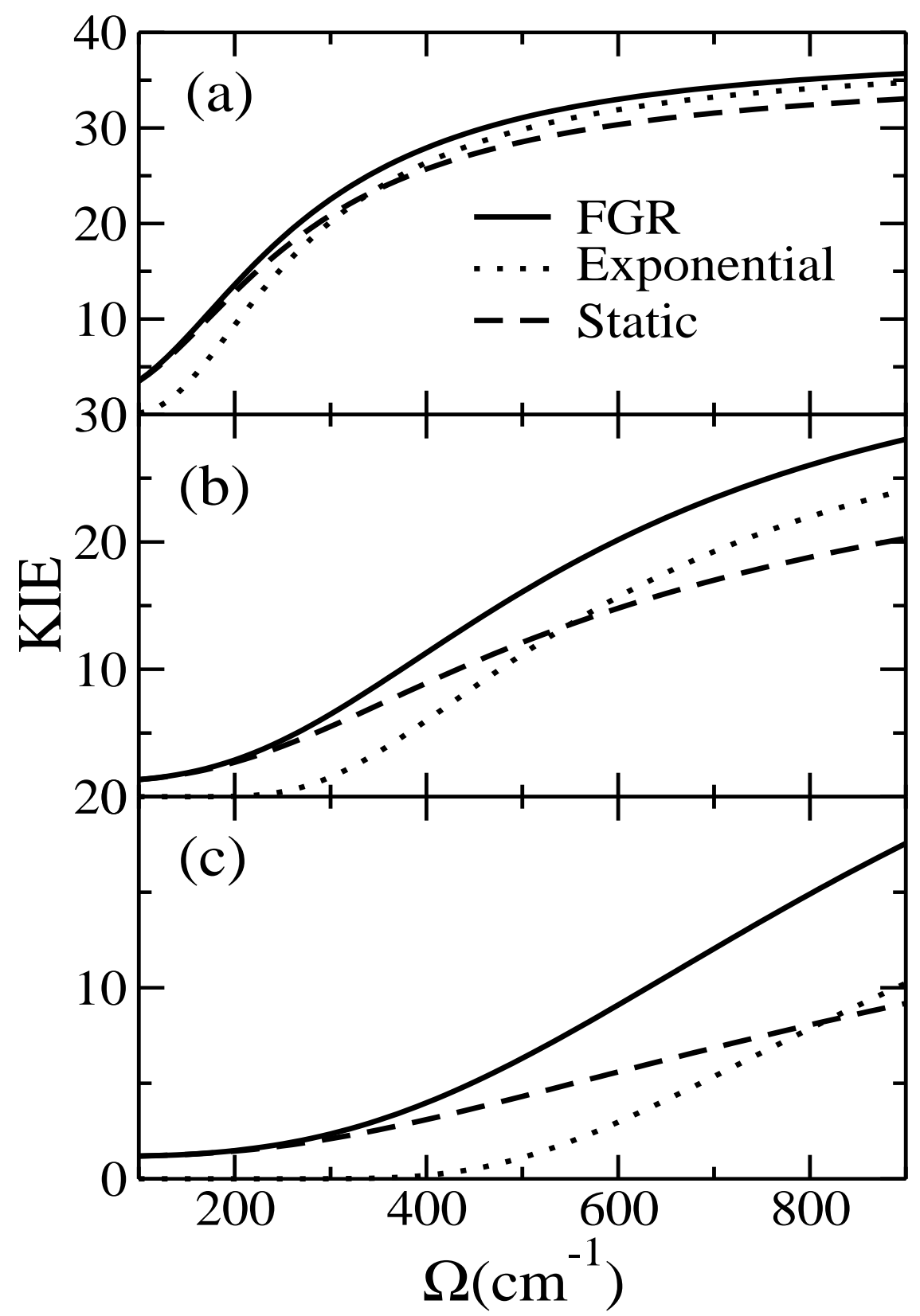

FIG. 4: KIE as a function of the proton donor-acceptor frequency $\Omega$. (a) $\mathrm{M}=100 \mathrm{amu}$, (b) $\mathrm{M}=$ $20 \mathrm{amu}$, (c) $\mathrm{M}=7 \mathrm{amu}$. The parameters are the same as in Fig. 3. 


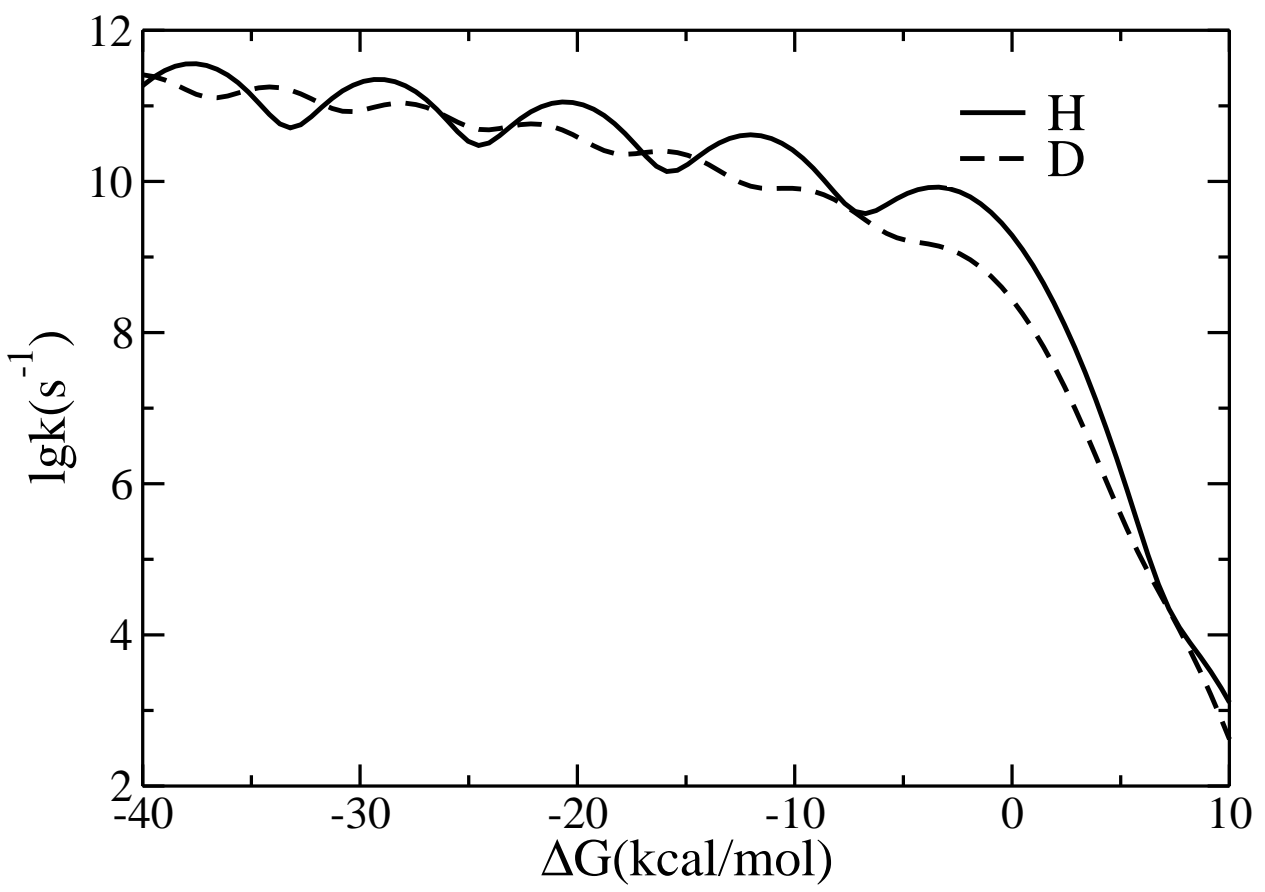

FIG. 5: PCET rate constants as a function of $\Delta G$ for small reorganization energy $\lambda=3 \mathrm{kcal} / \mathrm{mol}$. The other parameters are: $\Delta=100 \mathrm{~cm}^{-1}, M=20 \mathrm{amu}, \Omega=300 \mathrm{~cm}^{-1}, d=0.45 \AA$, and $T=300$ K. 


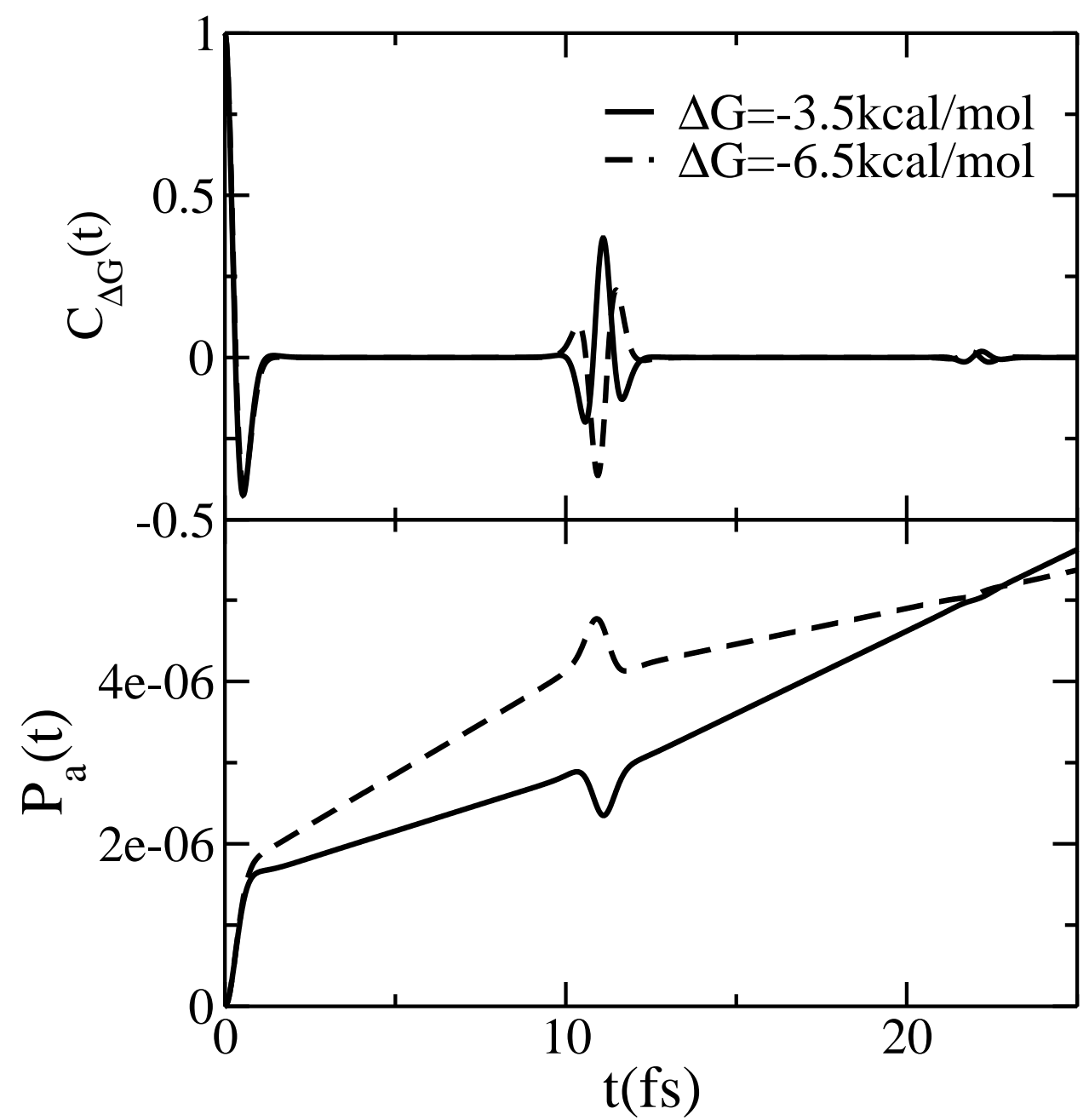

FIG. 6: (a) Normalized flux correlation function for $\Delta G=-3.5 \mathrm{kcal} / \mathrm{mol}$ (solid line) and -6.5 $\mathrm{kcal} / \mathrm{mol}$ (dashed line). (b) Population of the acceptor as a function of time. The other parameters are the same as in Fig. 5 . 


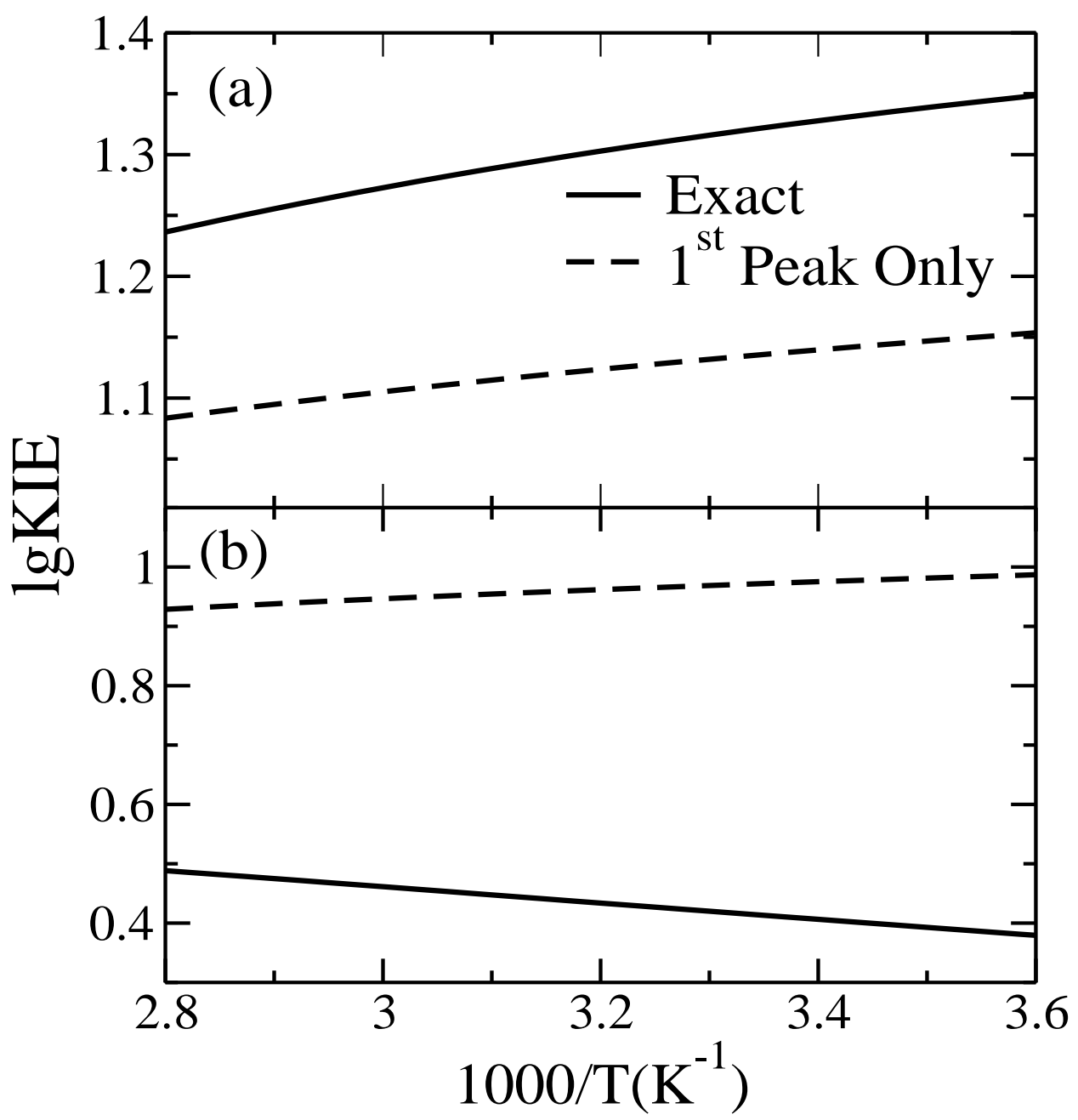

FIG. 7: KIE as a function of temperature for (a) $\Delta G=-3.5 \mathrm{kcal} / \mathrm{mol}$, and (b) $-6.5 \mathrm{kcal} / \mathrm{mol}$. Solid lines are the exact FGR result, while dashed lines show the results by including only the first peak of the correlation function as shown in Fig. 5 (a). The other parameters are the same as in Fig. 5 , 